\title{
Synchronization of symmetric chaotic systems
}

\author{
J. M. González-Miranda \\ Departamento de Física Fundamental, Universidad de Barcelona, Avenida Diagonal 647, 08028 Barcelona, Spain
}

(Received 10 January 1995; revised manuscript received 17 January 1996)

\begin{abstract}
This paper contains a study of the synchronization by homogeneous nonlinear driving of systems that are symmetric in phase space. The main consequence of this symmetry is the ability of the response to synchronize in more than just one way to the driving systems. These different forms of synchronization are to be understood as generalized synchronization states in which the motions of drive and response are in complete correlation, but the phase space distance between them does not converge to zero. In this case the synchronization phenomenon becomes enriched because there is multistability. As a consequence, there appear multiple basins of attraction and special responses to external noise. It is shown, by means of a computer simulation of various nonlinear systems, that: (i) the decay to the generalized synchronization states is exponential, (ii) the basins of attraction are symmetric, usually complicated, frequently fractal, and robust under the changes in the parameters, and (iii) the effect of external noise is to weaken the synchronization, and in some cases to produce jumps between the various synchronization states available. [S1063-651X(96)04806-4]
\end{abstract}

PACS number(s): 05.45.+b, 47.52.+j, 84.30. $-\mathrm{r}$

\section{INTRODUCTION}

It has been reported by Pecora and Carroll $[1,2]$ that two identical chaotic systems, characterized by exponential divergence of trajectories in phase space, may be synchronized. By synchronization they meant that the distance between the state points of both systems in phase space will converge to zero as time increases. This is achieved by driving one of the systems, the response, with a convenient chaotic signal generated by the other system, the drive. The possibility of realizing such synchronization has been successfully tested by computer simulations $[1-3]$ and experiments on electronic circuits $[4,5]$. This phenomenon is known as synchronization by homogeneous nonlinear driving. Besides its theoretical importance as a different phenomenon, there have been arguments on its possible practical interest in fields such as communications [6-9], control $[10,11]$, and neural science $[1,2,12]$.

Recent work in this field has drawn attention to the existence of meaningful generalizations of the idea of synchronization $[13,14]$. In these generalizations, the variables of the response evolve in correlation with the variables of the drive, although they do not take the same values. Amritkar and Gupte [13] characterize this generalization by means of convenient correlation dimensions that quantitatively measure the correlations between drive and response. Rulkov et al. [14] generalize the idea of synchronization equating variables from the response with a function of the variables of the drive.

In this article I will report on a particular type of nonlinear dynamical systems that exhibit synchronization behaviors of a generalized type together with the usual (Pecora and Carroll) synchronization. These nonlinear systems are described by equations that hold symmetries such that a response may synchronize in more than just one way to the drive. The synchronization behavior achieved is determined by the initial conditions of drive and response. In this case synchronization is to be understood in a generalized sense, as a state in which there is some generalized distance that goes to zero as time increases. This must be a nontrivial function of the phase space positions of drive and response. Then synchronization of chaotic systems by homogeneous nonlinear driving becomes a phenomenon richer than initially thought. In this article, I will develop these ideas for autonomous nonlinear systems and present a computer simulation study of various mathematical models whose equations exhibit symmetries of that type.

The contents of the article are arranged as follows. In Sec. II, I will present a general discussion on system symmetry, the appearance of multiple synchronization states, and its consequences. Moreover, I will present three particular clarifying examples. In Sec. III, I will present and discuss a numerical study of the model systems proposed in the preceding section as examples. In particular, I will pay attention to the chaotic behavior, the synchronization behavior, the basins of attraction to the several synchronization states available, and the effect of external noise on the synchronization. Finally, in Sec. IV, I will discuss and summarize the main results presented in the paper.

\section{THEORETICAL CONSIDERATIONS}

\section{A. Theory}

Let us begin with a brief review of some background concepts. The drive system is a homogeneous nonlinear autonomous $n$-dimensional system with variables $u=\left(u_{1}, \ldots, u_{n}\right)$, evolving under the equations $\dot{u}=f(u)$ with $f=\left(f_{1}(u), \ldots, f_{n}(u)\right)$, which can be divided in two subsystems $\nu=\left(u_{1}, \ldots, u_{m}\right)$ and $w=\left(u_{m+1}, \ldots, u_{n}\right)$, governed by the equations $\dot{\nu}=g(\nu, w)$ with $g=\left(f_{1}(u), \ldots, f_{m}(u)\right)$, and $\dot{w}=h(\nu, w)$ with $h=\left(f_{m+1}(u), \ldots, f_{n}(u)\right)$, respectively. The response system is a copy of $w, w^{\prime}=\left(u_{m+1}^{\prime}, \ldots, u_{n}^{\prime}\right)$, which evolves under $\dot{w}^{\prime}=h\left(\nu, w^{\prime}\right)$, so that it is run by its own variables $w^{\prime}=\left(u_{m+1}^{\prime}, \ldots, u_{n}^{\prime}\right)$ plus the variables $\nu=\left(u_{1}, \ldots, u_{m}\right)$ of the drive, which are called the drive signal. Pecora and Carroll define synchronization as the situation in which the distance in phase space between the sub- 
systems $w$ and $w^{\prime} \Delta w(t)=w^{\prime}(t)-w(t)$ converges to zero as time increases. The evolution of $\Delta w(t)$, in the limit of infinitesimal $\Delta w$, obeys the variational equation [1]: $d(\Delta w) / d t=D_{w} h(\nu, w) \Delta w$, where $D_{w} h(\nu, w)$ is the Jacobian of the $w$ subsystem. The Lyapunov exponents resulting from this equation are called conditional Lyapunov exponents and measure the average rate of divergence of $w^{\prime}$ from $w$ for small $\Delta w(t)$ [2]. The necessary and sufficient condition for synchronization is that the conditional Lyapunov exponents must be all negative [1,2]. In addition, Badola, Tambe, and Kulkarni [3], have reported that negativity of conditional Lyapunov exponents is only a guarantee of synchronization for a subset of initial conditions, so that only in these cases synchronization occurs. In particular, they reported computer simulation results of coupled lattice maps with negative conditional Lyapunov exponents, for which not all sets of initial conditions gave rise to synchronization.

My point in this paper is that the idea of synchronization can be enriched in a meaningful and interesting way. This happens when there is a transformation of coordinates $T: w \rightarrow w^{*}$, such that the evolution equations for $\nu$ and $w$ are invariant under this change, so that $\dot{\nu}=g\left(\nu, w^{*}\right)$ and $\dot{w}^{*}=h\left(\nu, w^{*}\right)$ hold. In this case, if there is a set of initial conditions for which $w^{\prime}$ synchronizes with $w$ in the sense that the distance $w^{\prime}-w \rightarrow 0$, then it must also happen that $w^{\prime *}-w \rightarrow 0$ for another set of initial conditions, obtained from the first by means of the above transformation of coordinates. However, the variables $w^{*}$ and $w$ can be obtained one from each other by means of some function relating them $w^{*}=T(w)$. Then, it follows that-for this second set of initial conditions-there is a generalized distance $D\left(w^{\prime}, w\right) \equiv T\left(w^{\prime}\right)-w$ that goes to zero as time increases. In a broader sense this means that $w^{\prime}$ is synchronized with $w$ in a form different than the usual $\left(w^{\prime}-w \rightarrow 0\right)$. So we have two different ways for the response to evolve in synchrony with the drive; that is, two different synchronization states available. Which of them is to be achieved in a particular case will depend on the initial conditions of drive and response in that case. It is important to note that the existence of this second synchronization state of the response follows from the symmetry of the system plus the existence of the usual synchronization state for a particular set of initial conditions. That is, besides symmetry one needs negative conditional Lyapunov exponents to ensure the usual synchronization state to exist [1], at least for a subset of initial conditions [3].

This opens up a scenario in which, for attractors which are invariant under $s$ nontrivial symmetry transformations $\left\{w_{1}^{*}=T_{1}(w), w_{2}^{*}=T_{2}(w), \ldots, w_{s}^{*}=T_{s}(w)\right\}$, one may have drive systems and signals for which there is a set of $s+1$ different synchronization states available, $\alpha \in\{0,1,2, \ldots, s\}$, each with its own distance $D_{\alpha}\left(w, w^{\prime}\right)=T_{\alpha}\left(w^{\prime}\right)-w$ that goes to zero for properly chosen initial conditions of drive and response. Here, $\alpha=0$ stands for the usual synchronization state $\left(T_{0}(w)=w\right)$. The synchronization states discussed here can be seen as particular cases of the idea of generalized synchronization as introduced by Rulkov et al. [14], who understand synchronization as equality of the variables of one of the subsystems to a function of the other subsystem. In the present case, the function $T_{\alpha}(w)$ plays this role, because $D_{\alpha} \rightarrow 0$ means $w=T_{\alpha}\left(w^{\prime}\right)$ for time large enough.
According to Badola, Tambe, and Kulkarni [3], when only the usual Pecora and Carroll synchronization state is available, the $2 n-m$ dimensional space of initial conditions $\left(u_{0}, w_{0}^{\prime}\right)$, is divided into two sets of initial conditions: one that leads to synchronization and another that does not. In the present case, the situation becomes even more interesting because the space of initial conditions is to be divided in as many subsets, or basins of attraction, as synchronization states available; to which, eventually, one must add possible initial conditions that do not drop down to synchronization states. From the above discussion it follows that such division in basins of attraction must exhibit symmetries reminiscent of those of the attractor. In particular, the regions of nonsynchronization initial conditions must be invariant under the coordinate transformations $T_{\alpha}$ of the equations of the drive; whereas, such kinds of transformations, must convert the different regions of generalized synchronization behavior into each other.

An important related issue is the effect of external perturbations, such as external noise in the synchronizing signal, on the synchronization phenomenon. In the present case, if the perturbations are not too large, one can expect their effect to be of one or both of the following types: (i) a weakening of the synchronization, in which none of the $D_{\alpha}$ converges to zero, but one of them remains small, so that its time average is a well defined small constant, and (ii) a jumping behavior, in which the response switches between the different synchronization (or nonsynchronization) states available. The particular effect observed and its intensity will depend on the system considered and on the strength and properties of the perturbation.

The weakening of the synchronization is mainly due to the competition between the tendency of the subsystems to synchronize, given by the conditional Lyapunov exponents, and the tendency of the noise to put them apart. Therefore one can expect different models to respond in the same qualitative manner. This behavior may be described by means of a set of probability densities for the distance $P_{\alpha}(D)$ each of them defined for one of the generalized distances involved. These probability densities are defined such that $P_{\alpha}(D) d D$ is the probability of finding the drive and response at a generalized distance $D_{\alpha}$ between $D$ and $D+d D$. In a weakly synchronization state, the generalized distance between drive and response will fluctuate in such a way that, for the value of $\alpha$ for which there is weak synchronization, the corresponding probability density $P_{\alpha}(D)$ will be peaked around a value of maximum expectation $\bar{D}$ which will be small and must increase with increasing noise strength. For the distances corresponding to values of $\alpha$ for which there is no synchronization at all, the corresponding distribution function should exhibit a broad maximum around values of the same magnitude than the attractor size. The value of $\bar{D}$ represents a compromise between the tendency of the distance to exponentially decay to zero (given by the conditional Lyapunov exponents), and the size and distribution of the external fluctuations that act to separate the response from the drive. As for the generalized distances that do not synchronize, they will take values corresponding to distances between different parts of the attractor; and, this is why, they should fluctuate around values of the same order of magnitude of the attractor size. 
Otherwise, the jumping behavior between synchronization states is very likely to be a consequence of the particular geometry of the attractors involved. A jump will occur when a fluctuation is large enough to send the system from one synchronization state to another. For a given distribution of fluctuations the probability of such an event will depend on how separated, in the response attractor, are the couples of state points $w^{\prime}$ and $w^{\prime *}$ which are related by a symmetry transformation $\left(w^{\prime *}\right)=T_{\alpha}\left(w^{\prime}\right)$. This is something given by the shape of the attractor. For example, attractors with the topology of a single loop (the type shown by the Rössler attractor [15]) are expected to be more robust against jumps than attractors with the topology of two tangent loops (the type of the Lorenz model [16]). This is because in this second case it is very likely that a fluctuation throws a system evolving in one loop to the other when it is moving in the neighborhood of the point of contact between the loops. This situations is, however much less likely in the case of the single loop because the distance between $w^{\prime}$ and $w^{\prime *}$ will be of the same order of magnitude that the attractor size. I shall remark at this point that I do not mean that the Rössler and Lorenz models are going to exhibit the behavior previously described. I am just using the overall image of two very well known dynamical systems to illustrate a general idea. Examples of systems that exhibit the behavior studied in this paper are presented in the next subsection.

\section{B. Examples}

Consider, for example, a drive with variables $u=(x, y, z)$ such that the equation $\dot{u}=f(u)$ has inversion symmetry in the $x-y$ plane; that is, it is invariant under the change $(x, y, z) \rightarrow(-x,-y, z)$. Let us take $z$ as the driving signal and $(x, y)$ as the response. If for some initial condition of the drive and the initial condition of the response $\left(x_{0}^{\prime}, y_{0}^{\prime}\right)$ it happens that $D_{0} \equiv\left|x^{\prime}-x\right|+\left|y^{\prime}-y\right| \rightarrow 0$, then $D_{1}$ $\equiv\left|x^{\prime}+x\right|+\left|y^{\prime}+y\right| \rightarrow 0$ must occur for the same initial condition of the drive and the initial condition of the response $\left(-x_{0}^{\prime},-y_{0}^{\prime}\right)$. So we will have two different synchronization states, each available from the proper set of initial conditions. A couple of particular examples of models holding this type of symmetry, to which I will dedicate particular attention in the numerical part of this paper, are given by the systems of first order differential equations

$$
\begin{gathered}
\dot{x}=-\alpha x+\beta z \sin (y), \\
\dot{y}=-y+(z-\gamma) x, \\
\dot{z}=1-x y,
\end{gathered}
$$

being $\alpha, \beta$, and $\gamma$ parameters of the model, and

$$
\begin{gathered}
\dot{x}=y+A \sin (\Omega y), \\
\dot{y}=-y-(z-R) x, \\
\dot{z}=x^{2}-z,
\end{gathered}
$$

with $A, \Omega$, and $R$ parameters of the model. These equations are invariant under the change $(x, y, z) \rightarrow(-x,-y, z)$. Then, if the drive $u=(x, y, z)$ is decomposed in $\nu=(z)$ and $w=(x, y)$, they have available two possible synchronization states, which correspond to the above distances $D_{0}$ and $D_{1}$.

As a second illustrative example consider the case of systems with variables $(x, y, z)$ that are invariant under rotations of the $x-y$ plane around the $z$ axis. In this case one will have as many synchronization states as the order of the rotational symmetry group $C_{m}$. A case of easy study is $C_{4}$; that is, invariance under rotations under angles that are integer multiples of $\pi / 2$ radians. In this case we will have invariance of the evolution equations under the identity, plus the following three different coordinate transformations: $T_{1}:(x, y, z)$ $\rightarrow(-x,-y, z), T_{2}:(x, y, z) \rightarrow(y,-x, z)$, and, $T_{3}:(x, y, z)$ $\rightarrow(-y, x, z)$. Therefore, given an initial condition of the drive, if $D_{0} \equiv\left|x^{\prime}-x\right|+\left|y^{\prime}-y\right| \rightarrow 0$ for some initial condition of the response $\left(x_{0}^{\prime}, y_{0}^{\prime}\right)$, then it must occur that $D_{1} \equiv\left|x^{\prime}+x\right|+\left|y^{\prime}+y\right| \rightarrow 0 \quad$ for $\quad\left(-x_{0}^{\prime},-y_{0}^{\prime}\right), \quad D_{2}$ $\equiv\left|y^{\prime}-x\right|+\left|x^{\prime}+y\right| \rightarrow 0 \quad$ for $\quad\left(y_{0}^{\prime},-x_{0}^{\prime}\right)$, and $D_{3}$ $\equiv\left|y^{\prime}+x\right|+\left|x^{\prime}-y\right| \rightarrow 0$ for $\left(-y_{0}^{\prime}, x_{0}^{\prime}\right)$. Then we will have four different synchronization states available from the proper sets of initial conditions. An example of an attractor that exhibits $C_{4}$ symmetry, and which will be studied below in this paper, is given by the following three dimensional flux:

$$
\begin{gathered}
\dot{x}=\epsilon(x, y)[-\alpha x+\beta z \sin (y)]+\delta(x, y)[-x+(\gamma-z) y], \\
\dot{y}=\epsilon(x, y)[-y+(z-\gamma) x]+\delta(x, y)[-\alpha y-\beta z \sin (x)], \\
\dot{z}=\epsilon(x, y)[1-x y]+\delta(x, y)[1+x y],
\end{gathered}
$$

being $\quad \epsilon(x, y)=[1+\tanh (2 \sigma x y)] / 2, \quad \delta(x, y)=[1$ $-\tanh (2 \sigma x y)] / 2$, and $\alpha, \beta, \gamma$, and $\sigma$ are parameters of the model.

I must note that the equations presented above are for mathematical models, with no immediate physical interpretation or applied motivation, that are proposed just for the sake of illustration. In fact, Eqs. (1) and (3) are modifications of the equations for a magneto-mechanical model proposed by Rikitake to study the time reversal of earth's magnetic field [17], and Eqs. (2) are a mathematical model introduced
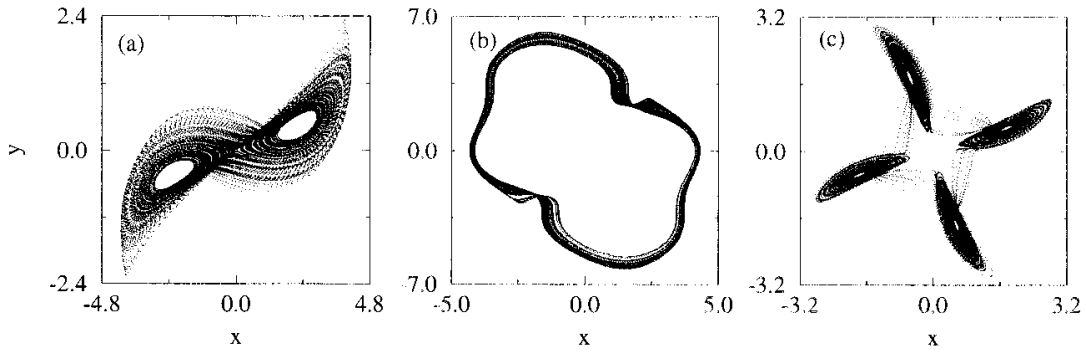

FIG. 1. Projections of the attractors defined by the proper evolution equations onto the $(x-y)$ plane for the three nonlinear systems described in the text: (a) A1, (b) A2, and (c) A3. 

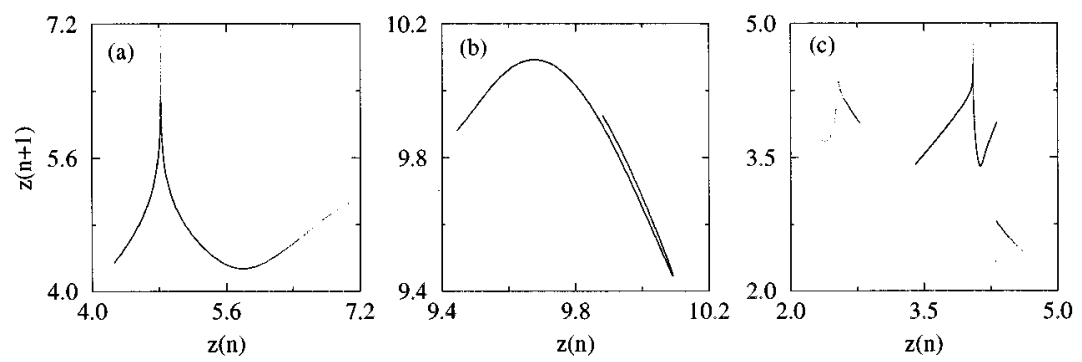

FIG. 2. Return maps for the three symmetric nonlinear systems studied: (a) A1, (b) A2, and (c) A3.

here only as an illustrative example. However, when dealing with models with direct physical motivation, the variables $x$, $y$, and $z$ will be physical quantities whose values define a particular state of the system. In particular, they may be velocities, electrical currents, temperature gradients, or other physical quantities, so that a change of sign or numerical values would mean significant changes in the corresponding experimental situations. Accordingly, the occurrence of $D_{\alpha \neq 0} \rightarrow 0$ instead of $D_{0} \rightarrow 0$ would imply different physical situations, and so it follows that what I am describing here may manifest itself as a phenomenon with physical meaning.

\section{NUMERICAL EXPERIMENTS}

\section{A. Chaotic attractors}

To illustrate the theoretical points raised above I will now present and discuss some numerical results for the mathematical models used above as examples. These have been obtained working in double precision, and integrating the evolution equations by means of a fourth order Runge-Kutta algorithm. Most of the numerical results in this section correspond to the following cases: (A1) Equation (1), at the parameter values $\alpha=1.5, \beta=2.0$, and $\gamma=3.75$, and an integration time step of 0.03 , (A2) Equation (2), at parameter values $A=3.2, \Omega=1.4$, and $R=5.2$, and an integration step of 0.01, (A3) Equation (3) at parameter values $\alpha=2.0, \beta=2.0$, $\gamma=3.0$, and $\sigma=16.0$, and an integration step of 0.03 . These cases will be designated throughout this paper with the corresponding abbreviation An, $n \in\{1,2,3\}$.

In the first place, to ensure that at the above parameter values the systems considered are chaotic, I have performed a series of standard tests $[18,19]$. The results of some of them are displayed in Figs. 1-3. In Fig. 1 there appear plots of the attractors which tend to fill up a section of the phase space, as corresponds to chaotic evolutions. Besides, this figure shows how the attractors exhibit the symmetry of the corresponding evolution equations. In particular, one can appreci-
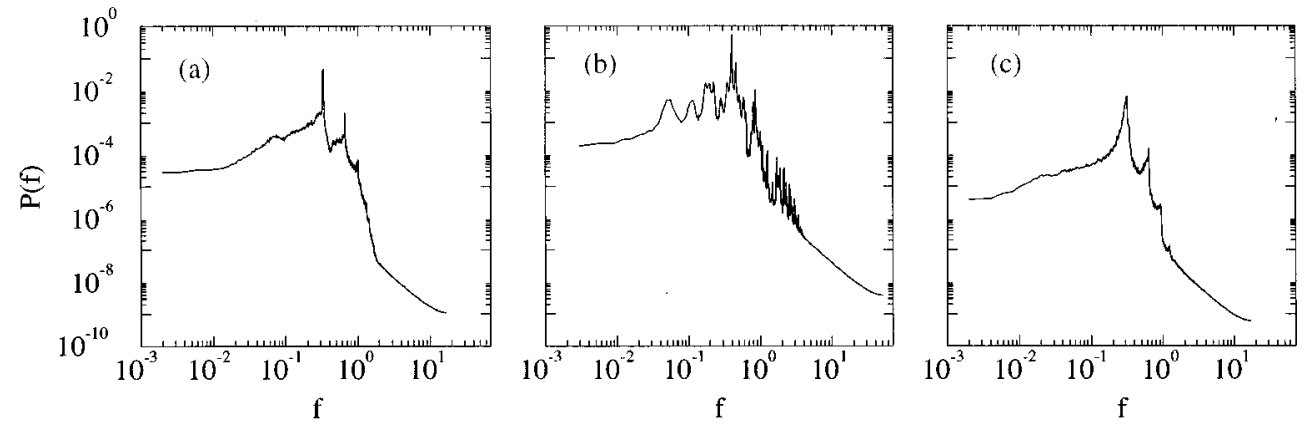

FIG. 3. Power spectra for the $z$ signal for the three symmetric nonlinear systems studied: (a) A1, (b) A2, and (c) A3. ate how A1 and A2, despite showing the same type of symmetry, present a different topology, i.e., A1 is a double loop, while A2 is a single loop. In Fig. 2, there appear plots of the $(n+1)$ th maximum of $z$ versus the $n$th for sets of 10000 points. This shows the existence of approximately quadratic or tent maps embedded in the attractors, which is clear evidence of chaos. Additional evidence is given by the power spectrum of the variables $x(t), y(t)$, and $z(t)$ which exhibit the presence of a broad spectrum of frequencies as shown, for example, in Fig. 3 for the variable $z(t)$. Moreover, I have computed the Lyapunov spectrum of the systems by means of the technique developed independently by Benettin et al. [20], and by Shimada and Nagashima [21]. The results obtained are displayed in Table I. Because the theorem of Haken [22], on the existence of at least one vanishing Lyapunov exponent, does hold in this case, these are to be understood as chaotic spectra of the type $(+, 0,-)$.

\section{B. Synchronization behavior}

Let us now turn to the synchronization behavior of these system under $z$ driving. First of all, I have computed the conditional Lyapunov exponents using the Benettin et al. [20], and Shimada and Nagashima [21] method. The results obtained appear in Table I. In all cases I have found that both conditional Lyapunov exponents are negative; so, the Pecora and Carroll condition for synchronization is satisfied. Then one can expect, for all three attractors, to exist a set of initial conditions, $\left(x_{0}, y_{0}, z_{0}, x_{0}^{\prime}, y_{0}^{\prime}\right)$, from which the response synchronizes with the drive in the sense that $D_{0} \rightarrow 0$. According to the discussion in the preceding section, this implies that we should observe generalized synchronization behaviors of the proper type $D_{\alpha} \rightarrow 0$, for the initial conditions of the response obtained from the above $\left(x_{0}^{\prime}, y_{0}^{\prime}\right)$ by means of the coordinate transformation $T_{\alpha}$. The discussion of the results obtained for the different cases studied follows.

For A1, I have numerically integrated the fifth order nonlinear system defined by equations 
TABLE I. Spectra of Lyapunov exponents $\left(\lambda_{1}, \lambda_{2}, \lambda_{3}\right)$ and conditional Lyapunov exponents under $z$ driving $\left(\lambda_{1}^{(z)}, \lambda_{2}^{(z)}\right)$ for the three dynamical systems studied in this article.

\begin{tabular}{ccc}
\hline \hline & $\left(\lambda_{1}, \lambda_{2}, \lambda_{3}\right)$ & $\left(\lambda_{1}^{(z)}, \lambda_{2}^{(z)}\right)$ \\
\hline$A 1$ & $(0.232,0.000,-2.732)$ & $(-0.104,-2.396)$ \\
$A 2$ & $(0.126,0.000,-2.126)$ & $(-1.000,-1.000)$ \\
$A 3$ & $(0.136,0.000,-2.981)$ & $(-0.053,-2.790)$ \\
\hline \hline
\end{tabular}

$$
\begin{gathered}
\dot{x}^{\prime}=-\alpha x^{\prime}+\beta z \sin \left(y^{\prime}\right), \\
\dot{y}^{\prime}=-y^{\prime}+(z-\gamma) x^{\prime},
\end{gathered}
$$

combined with Eq. (1). In this case, the two types of synchronization behaviors mentioned above $D_{0} \rightarrow 0$ and $D_{1} \rightarrow 0$, can be obtained from the proper initial conditions. As an example, the time evolution of $D_{0}(t)$ and $D_{1}(t)$ is displayed in Fig. 4(a) for an initial condition in the basin of attraction for the $\alpha=1$ generalized synchronization state. The evolution of $D_{1}(t)$ shows an oscillatory exponential decay to zero, while $D_{0}(t)$ oscillates around a constant value, with a magnitude around the size of the attractor. A plot of $D_{0}(t)$ and $D_{1}(t)$, for initial conditions leading to the usual synchronization state, would look like that in Fig. 4(a) but with the curves for $D_{0}(t)$ and $D_{1}(t)$ swapped.

For A2, I have integrated the fifth order nonlinear system defined by equations

$$
\begin{aligned}
& \dot{x}^{\prime}=y^{\prime}+A \sin \left(\Omega y^{\prime}\right), \\
& \dot{y}^{\prime}=-y^{\prime}-(z-R) x^{\prime},
\end{aligned}
$$

combined with Eq. (2). In this case, I have observed that not all initial conditions of drive and response give rise to evo- lutions towards one of the two synchronization states described above. In fact, I was able to observe four different behaviors available to the response from the appropriate sets of initial conditions: (1) synchronization of the $D_{0} \rightarrow 0$ type, (2) synchronization of the $D_{1} \rightarrow 0$ type, (3) a nonsynchronized bounded state in which the response moves in an ample orbit around the drive, never intersecting it, and (4) a nonsynchronized unbounded state, in which the response follows a divergent evolution, which slowly spirals outward around the drive so that both distances $D_{0}(t)$ and $D_{1}(t)$ diverge to infinity. These different behaviors are illustrated in Fig. 4(b), were $D_{1}(t)$ is displayed as a function of time $t$ for four different initial conditions leading each to one of the four types of behavior available. For an initial condition in the $D_{1} \rightarrow 0$ basin of attraction the $D_{1}(t)$ function decays in an oscillatory exponential way to zero (curve 2). For the nonsynchronized unbounded case an oscillatory exponential divergence is clearly seen (curve 4). For the other two cases (curves 1 and 3) the generalized distance fluctuates around an average finite value which is larger in the nonsynchronized bounded case (curve 3). A similar plot, with the behavior for the cases 1 and 2 swapped, would be observed if the distance $D_{0}(t)$ were shown. For additional illustration a parametric plot of $x^{\prime}(t)$ versus $x(t)$ is displayed in Fig. 4(c) for the nonsynchronized bounded case. This type of plot, when synchronization occurs, looks like a straight line segment with a slope equal to 1 for the $D_{0} \rightarrow 0$ case, and equal to -1 for the $D_{1} \rightarrow 0$ case. The wide dispersion of points in Fig. 4(c) is indication of no correlation between the signals. The overall inclination of the cloud of points, with a slope approximately equal to 3 , indicates that the orbit for the response is wider that the orbit for the drive. A plot of $y^{\prime}(t)$ versus $y(t)$ would be similar to that in Fig. 4(c).

For A3, I have numerically integrated the fifth order nonlinear system defined by equations
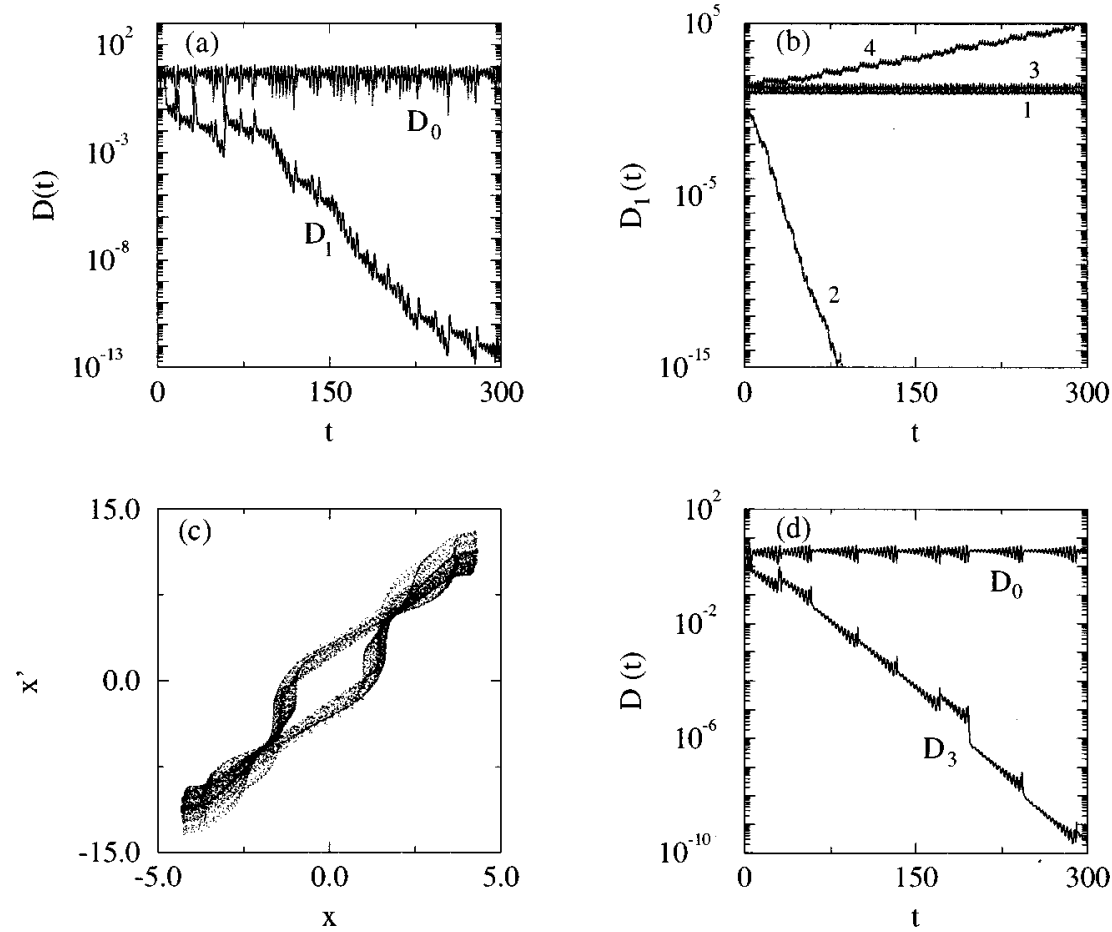

FIG. 4. (a) Time evolution of the generalized distances $D_{0}(t)$ and $D_{1}(t)$ for an initial condition in the $D_{1} \rightarrow 0$ basin of attraction for attractor A1. (b) Time evolution of $D_{1}(t)$ for the four different behaviors available to the response in the case of attractor A2: (1) synchronization of the $D_{0} \rightarrow 0$ type, (2) synchronization of the $D_{1} \rightarrow 0$ type, (3) nonsynchronized bounded state, and (4) nonsynchronized unbounded state. (c) Parametric plot of $x^{\prime}$ versus $x$ in the nonsynchronized bounded state. (d) Time evolution of the generalized distances $D_{0}(t)$ and $D_{3}(t)$ for an initial condition in the $D_{3} \rightarrow 0$ basin of attraction for attractor A3. 
(a)

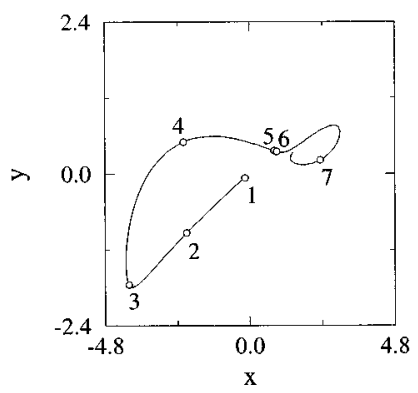

(d)
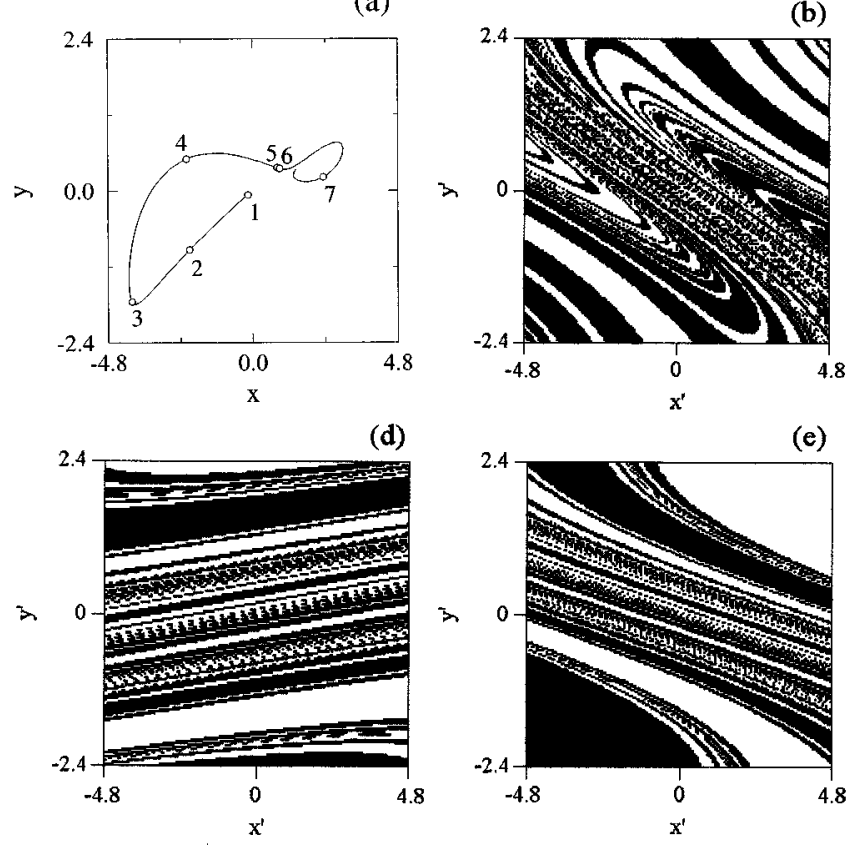

\section{.}

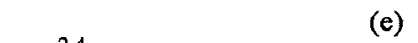

(e)

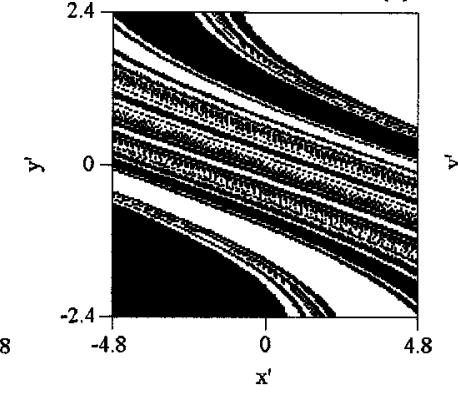

$$
\begin{aligned}
\dot{x}^{\prime}= & \epsilon\left(x^{\prime}, y^{\prime}\right)\left[-\alpha x^{\prime}+\beta z \sin \left(y^{\prime}\right)\right]+\delta\left(x^{\prime}, y^{\prime}\right) \\
& \times\left[-x^{\prime}+(\gamma-z) y^{\prime}\right], \\
\dot{y}^{\prime}= & \epsilon\left(x^{\prime}, y^{\prime}\right)\left[-y^{\prime}+(z-\gamma) x^{\prime}\right]+\delta\left(x^{\prime}, y^{\prime}\right) \\
& \times\left[-\alpha y^{\prime}-\beta z \sin \left(x^{\prime}\right)\right],
\end{aligned}
$$

combined with Eq. (3). As in the previous cases, I have been able to observe all the synchronization states given by $D_{\alpha}(t)$, with $\alpha \in\{0,1,2,3\}$ for initial conditions chosen conveniently (see Sec. II B). As an example, a plot of $D_{3}(t)$ displaying the characteristic exponential decay to zero appears in Fig. 4(d), for an initial condition in the $D_{3} \rightarrow 0$ basin of attraction. Moreover, in this plot there appears $D_{0}(t)$, for the same initial condition, which fluctuates around a finite value [the same behavior will be observed if $D_{1}(t)$ or $D_{2}(t)$ were shown]. The corresponding plots for the other three cases would exhibit a similar look. I must note that besides these four synchronization behaviors available, there is the possibility of no synchronization between drive and response for initial conditions of the response close to $(0,0)$. In this case the response evolves towards a fixed point located at the origin of coordinates.

The plots for $D_{\alpha}(t)$ in Fig. 4 illustrate the possibility of the synchronization states of types different than the usual,
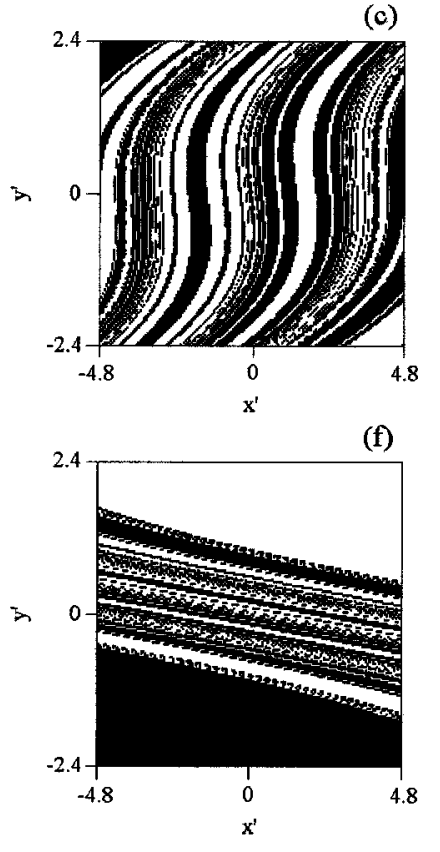

(f)

FIG. 5. (a) Drive attractor trajectory, for attractor A1, with indication of the points taken as initial conditions for the calculation of the basins of attraction depicted in this and the following two figures. Plots of the basins of attraction of the response to the different behaviors allowed to it when $z$ driving is applied: (b) point 1 , (c) point 3 , (d) point 4 , (e) point 5 , and (f) point 7.

described in Sec. II, to exist. It is worth noting that Pecora and Carroll also reported this type of oscillatory exponential decay in their study of synchronization of systems with just the usual synchronization state available [1,2]. The observation of systems with negative conditional Lyapunov exponents for which some initial conditions synchronize while others do not, gives additional illustration to the finding of Badola, Tambe, and Kulkarni [3] that negativity of conditional Lyapunov exponents is not a guarantee of synchronization for all initial conditions.

\section{Basins of attraction}

The study of the basins of attraction in the present case is quite difficult, because we are faced with a five dimensional space of initial conditions. To have some insight on the shape of the basins of attraction to the different types of behaviors available to the response, I have proceeded as follows. I chose some point in the attractor as initial condition for the drive, $\left(x_{0}, y_{0}, z_{0}\right)$, and a grid of $N \times N$ initial points in the $\left(x^{\prime}, y^{\prime}\right)$ subspace for the response. Then, I ran the equations for drive and response to see the time evolution of $D_{\alpha}$ for each point in the grid. A plot was prepared in which each point of the grid is colored according to the synchronization state achieved, so that one can have an image of the basins of

(a)

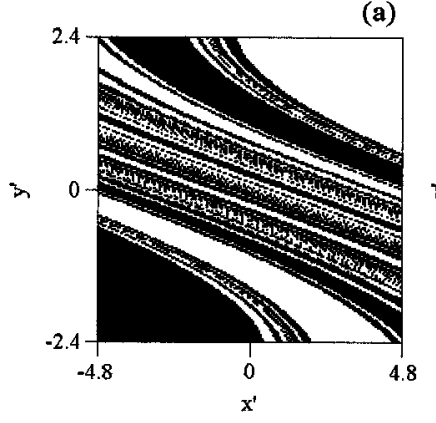

(b)

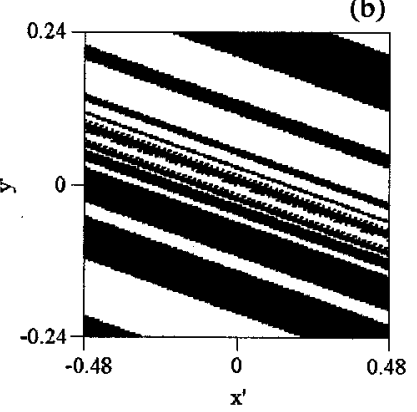

(c)

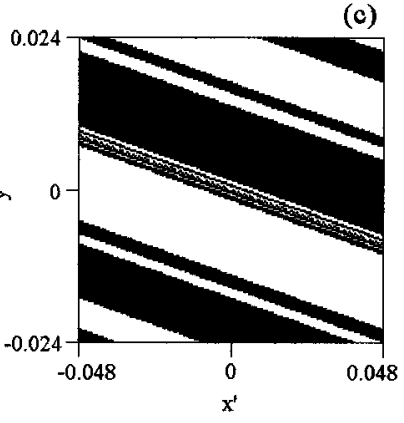

FIG. 6. Plots of successive amplifications of a basin of attraction of attractor A1. The initial condition for the drive point is given by point 6 in Fig. 5(a), the origin of coordinates is always at the center of the image, and the amplification factors are: (a) $\times 1$, (b) $\times 10$, and (c) $\times 100$. 
(a)
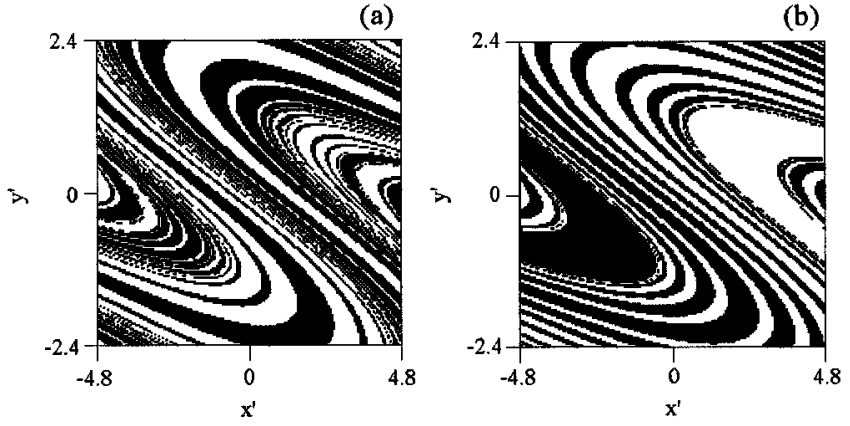

attraction for the particular initial condition of the drive $\left(x_{0}, y_{0}, z_{0}\right)$. In almost all the cases that follow, these pictures are centered at the origin of coordinates and show a region of phase space a bit larger than the one spanned by the attractor. In the case when the pictures represent regions not centered at the origin or with dimensions different than those of the drive attractor this is explicitly stated. In this study, I have paid particular attention to the evolution of the basins with the values of $\left(x_{0}, y_{0}, z_{0}\right)$ (always chosen as a point in the stable drive attractor), to the nature of the basin boundaries, and to the effect of the changes in the parameters of the system on the basin pictures. In this last case I have studied attractors with parameters different than those indicated at the beginning of this section, chosen so that the system is still chaotic, and the conditional Lyapunov exponents continue to be negative. In this case, the values of the drive initial conditions $\left(x_{0}, y_{0}, z_{0}\right)$ cannot be exactly the same for all choices of parameters because they are to be points of the stable attractor which is a set of points that changes when the parameters change. However they have been carefully chosen to ensure that the points used for every choice of parameters are very close among them.

Representative examples of such basins in the case of A1 are displayed in Figs. 5, 6, and 7. There, grids of $161 \times 161$ initial conditions for the response are displayed with the points in the grid colored white if $D_{1} \rightarrow 0$ and black if $D_{0} \rightarrow 0$. Except when explicitly indicated, the regions studied has a size of $9.6 \times 4.8$; which corresponds to the region of phase space shown in Fig. 5(a). All initial conditions give rise to one of the two synchronization states available, with the only exception of the origin of coordinates, which is an unstable fixed point. Moreover, from these pictures it is clear that if $D_{0} \rightarrow 0$ for $\left(x_{0}^{\prime}, y_{0}^{\prime}\right)$, then $D_{1} \rightarrow 0$ for $\left(-x_{0}^{\prime},-y_{0}^{\prime}\right)$, as expected. The basin shapes vary with the initial condition of the drive. This variation is illustrated in Fig. 5, where five basin pictures are shown with an indication of the drive point trajectory to which they belong. Additional pictures can be seen in Fig. 6(a) and Fig. 7(a). The shape of the basins evolves smoothly along the drive trajectory [compare Fig. 5(e) with Fig. 6(a)] but experiences noticeable changes when the point in the drive attractor are far away. In general, the shapes of the basins are complicated and the two types of synchronization behaviors available appear entangled. The nature of these basins seems to be fractal as more detail is seen with amplification; as illustrated in Fig. 6, where successive amplifications by a power of ten of the central region of one of them are displayed. Moreover, as illustrated in Fig. 7 , the overall shape of the basin pictures appears robust under changes in the parameters of the attractor; although, the

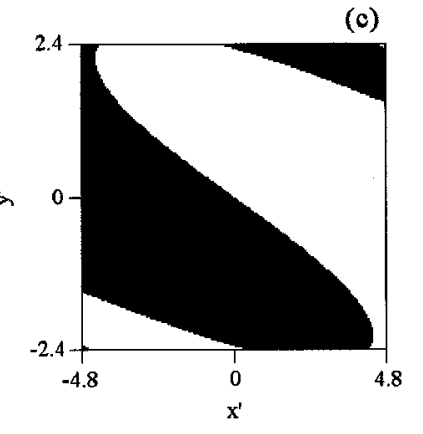

(c)

details of the different pictures change with changing parameters. In this figure, the shapes of the basins for close sets of values of the drive initial condition $\left(x_{0}, y_{0}, z_{0}\right)$ and different values of the parameter $\alpha \in\{1.5,1.6,2.0\}$ appear. In particular it is worth noting that for $\alpha=2.0$ the basin boundaries become smooth and simple.

For A2, representative examples of such basins are shown in Figs. 8-11, where the grid of initial conditions for the drive is displayed with the points in the grid colored white if $D_{1} \rightarrow 0$, black if $D_{0} \rightarrow 0$, and gray if none of these events happen. A small white dot indicates the position of the initial condition of the drive. The grids of initial conditions for the response displayed contain $239 \times 239$ points. Except when indicated, the region studied has a size of $10 \times 14$ and corresponds to the phase space region shown in Fig. 8(a). In all plots the origin of coordinates is at the center of the image, and the expected symmetry of the basins pictures is obvious. The particular shape of the pattern observed depends on the set of initial conditions chosen for the drive, as illustrated in Fig. 8, Fig. 9(a), and Fig. 10(a). As in the previous case, one (a)

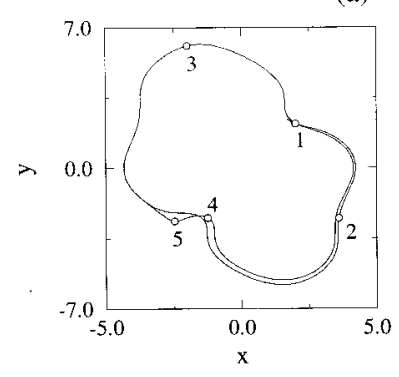

(c)

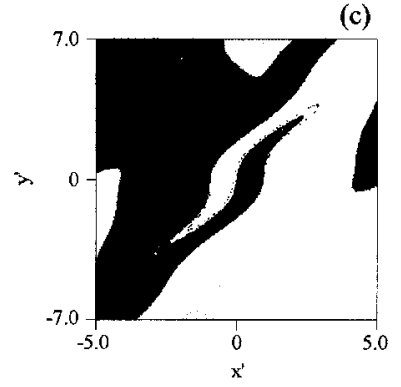

(b)

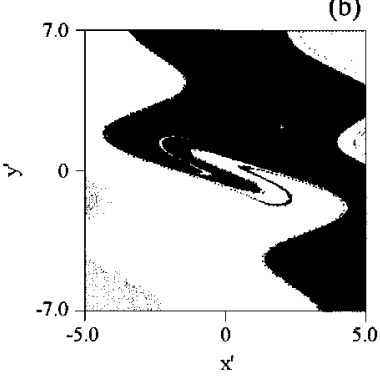

(d)

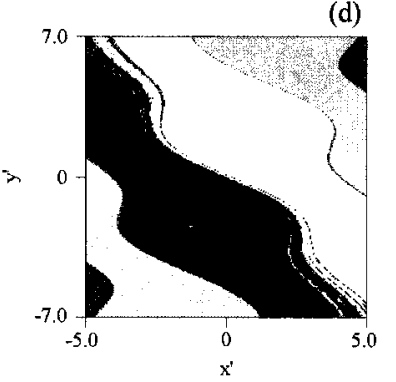

FIG. 8. (a) Drive attractor trajectory, for A2, with indication of the points taken as initial conditions for the calculation of the basins of attraction depicted in this and Figs. 9, 10 and 11. Plots of the basins of attraction of the response to the different behaviors allowed to it when $z$ driving is applied: (b) point 1, (c) point 3, and (d) point 4 . 
(a)

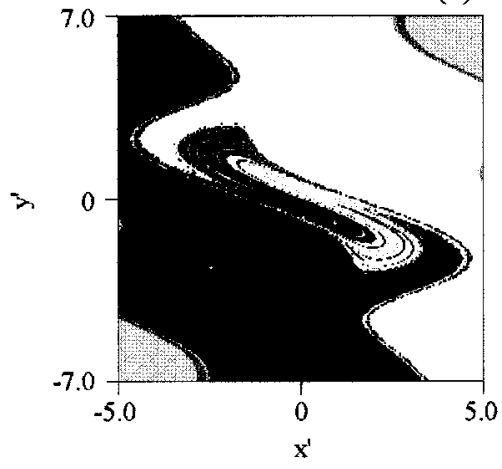

(b)

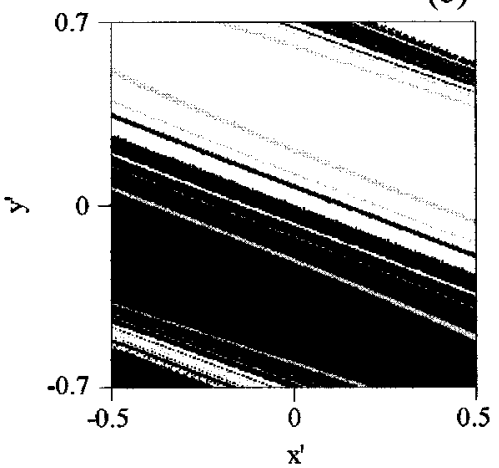

(c)

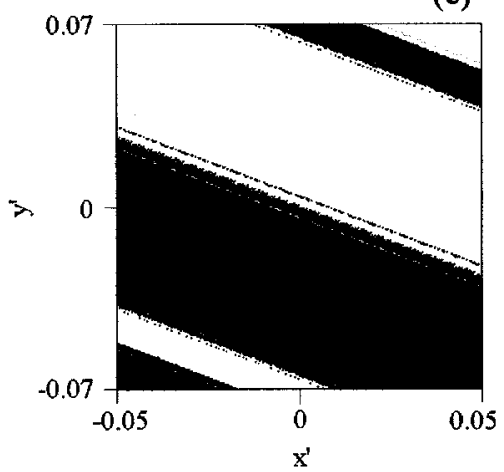

FIG. 9. Plots of successive amplifications of the basin of attraction of the response to the different behaviors allowed to the A2 attractor. The initial condition for the drive point is given by point 5 in Fig. 8(a), all the plots are centered at the origin of coordinates, and the amplification factors are: (a) $\times 1$, (b) $\times 10$, and (c) $\times 100$.

obtains an evolution of the basin pictures with the change on the drive initial condition $\left(x_{0}, y_{0}, z_{0}\right)$. However the overall appearance of the basins is quite different than those of A1. The more significant difference is that, despite the variety of behaviors available, the different basins of attraction are wide and clearly separated. Moreover, the values of the initial conditions of the response coincident with those of the drive always appear far from the basin boundaries. These basin boundaries, however, still seem to be fractal as illustrated in Fig. 9 by means of successive amplification, by a factor ten, of the central region of one of them. The basin shapes appear robust under the changes in parameter values, as shown in Fig. 10, where basin pictures are displayed for close drive initial conditions corresponding to different values of the parameter $R \in\{5.20,5.15,4.80\}$, showing the same overall shape but changes in the details. A particularity of this system is the existence of other states available to the response besides to the two synchronized ones. To show more clearly the presence of these states, in Fig. 11, there are displayed basin pictures for the same drive initial conditions as those in Fig. 8, but displaying a region of $100 \times 140$ (i.e., 10 times larger). The light gray region indicates the initial conditions that gives rise to bounded nonsynchronized states, and the medium gray regions (far from where the attractor evolves) the initial condition from which the distances diverge.
Results for the basins of attraction of A3, are displayed in Figs. 12-15. As for A1, the grids of points are $161 \times 161$. Different shades of gray are used in this case with the following code: black for $D_{0} \rightarrow 0$, white for $D_{1} \rightarrow 0$, light gray for $D_{2} \rightarrow 0$, dark gray for $D_{3} \rightarrow 0$, and medium gray for the initial conditions from which the response drops towards the fixed point at the origin. Moreover, a white dot indicates the position of the initial condition of the drive. The region studied, except when indicated, has a size of $6.4 \times 6.4$ and corresponds to the square shown in Fig. 12(a). The symmetries associated to the $C_{4}$ symmetry of the attractor are clearly seen through these pictures, except in Figs. 13(b) and 13(c) that are not centered at the origin of coordinates. The evolution of the basins with the initial conditions, which is smooth as in the previous cases, is illustrated in Fig. 12, and Figs. 13(a) and 14(a). Despite having its own shape, these basins resemble those of $\mathrm{A} 2$ in that there are wide regions for each of the behaviors available in which there is no mixing among the different behaviors available. This is so despite the basin boundaries seeming to be fractal as illustrated in Fig. 13 by successive amplifications by a power of ten of the central region of one of them. The changes with the parameters of the system resemble those of the two previous cases in that the overall shape of the basins does not change, while the details of the boundaries are somewhat modified. This is shown in Fig. 14 where there appear results for the parameter (a)

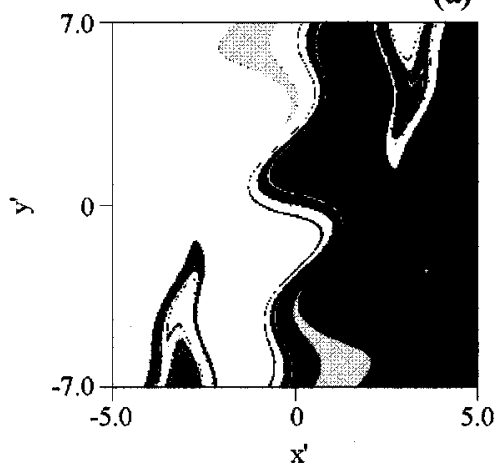

(b)

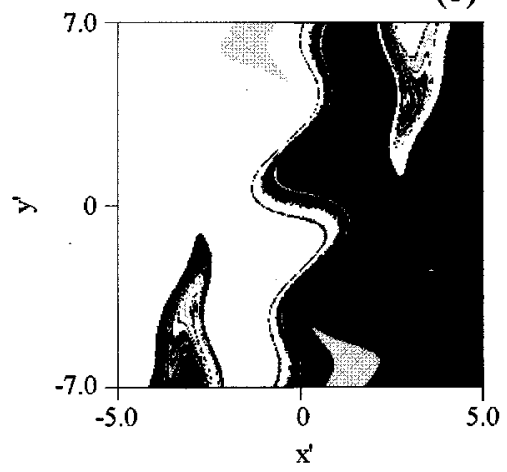

(c)

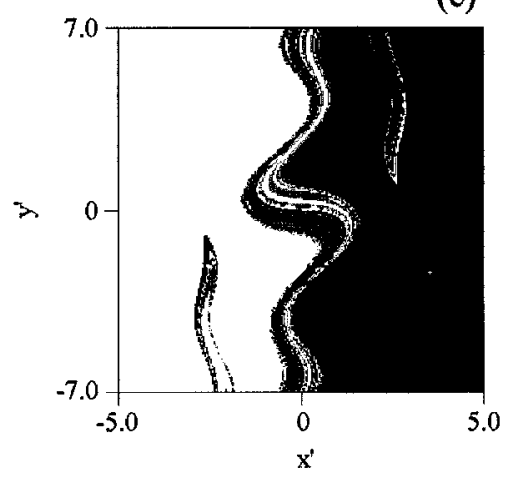

FIG. 10. Plots of the basin of attraction corresponding to the attractor A2 for almost the same initial condition of the drive [point 2 of Fig. $8(\mathrm{a})]$, and different values of the parameter $R$ : (a) 5.20 , (b) 5.15 , and (c) 4.80 . 
(a)

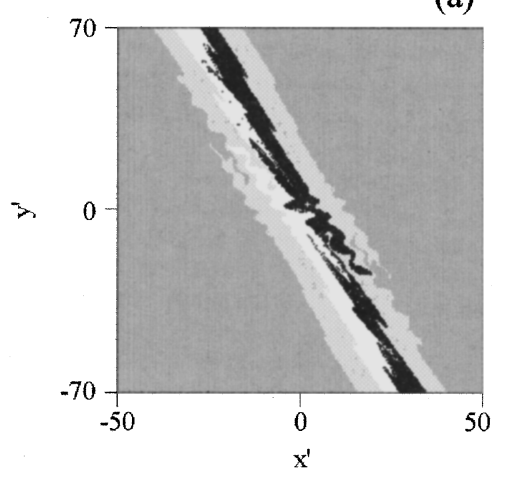

(b)

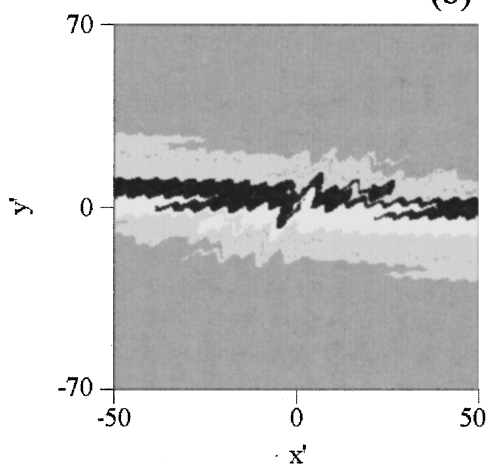

(c)

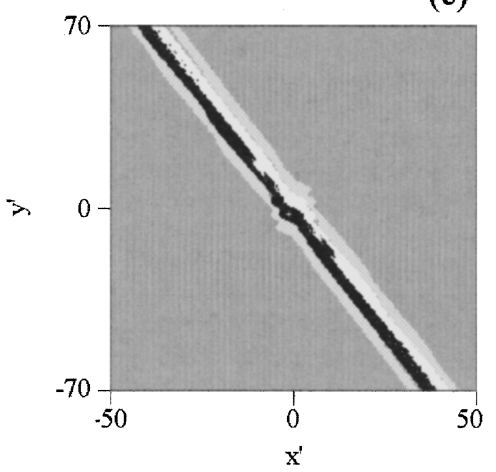

FIG. 11. Basins of attraction for the same cases shown in Fig. 8, but showing a region 10 times wider: (a) point 1, (b) point 3, and (c) point 4.

$\sigma$ taking values in $\{16.0,14.0,12.0\}$. An interesting observation, for this attractor, is that, although almost all the time the numerical values corresponding to the initial condition of the attractor are well inside the $D_{0} \rightarrow 0$ basin of attraction, for some special points they become very close to the boundaries. This situations is illustrated in Fig. 15, where there appear the boundaries for two close points near a region in which the attractor bifurcates between two different loops. It deserves to be noted how different the basins are, despite points 1 and 2 being quite close. This reflects the fact that point 1 is in a trajectory inside a loop, while point 2 is in a trajectory corresponding to a jump between loops.

The results in Figs. 5 to 15 illustrate the practical possibility of synchronization in a way different than usual when we deal with symmetric systems. Moreover, they tell us that each particular nonlinear system will display a set of peculiar patterns that are a specific property of that system. This means that the shapes of the basin boundaries are expected to be as diverse as the dynamical systems themselves are. However, some regularities are present: (i) the basins display symmetries reminiscent of those of the dynamical system to which they belong, (ii) they change smoothly with the variation of $\left(x_{0}, y_{0}, z_{0}\right)$ along the drive trajectory, and (iii) they appear to be robust under small changes of the parameters of the system, despite that a change in parameters implies changes in the attractor properties (expected to be small) as well as in the values of the initial conditions of the drive. Large changes in the parameters can alter in a significant extent the basin boundaries. Moreover, the boundary basins seem to be fractal, although this is not expected to be the general rule as some of the plots indicate [see Fig. 7(c) and Fig. 14(c)].

\section{Effect of external noise}

To test the effect of external noise on the synchronization in the models studied here, I have studied how synchronization is affected by a white noise in the driving signal. To do this I have integrated, together with the equations for the drive, the corresponding equations for the response adding a random Gaussian variable $\eta$ with a standard deviation $\sigma$, to the drive signal $z$. In the calculations performed, I have chosen initial conditions well inside one of the basins of attraction to one of the synchronization states involved. Then, I ran the equations of motion for $10^{8}$ time steps, for values of $\sigma$ between 10 and $1.0 \times 10^{-5}$ for $\mathrm{A} 1,10$ and $5.0 \times 10^{-4}$ for $\mathrm{A} 2$, and 10 and $1.0 \times 10^{-8}$ for A3. The objective is to get some insight on the shape and behavior of the probability densities $P_{\alpha}(D)$ and the rate of jumps between synchronization states. To achieve this, I have computed, in that time window: (i) the histograms for the number of occurrences $\Delta N_{\alpha}(D)$ of a generalized distance $D_{\alpha}$ between $D$ and $D+\Delta D$, and (ii) the number of jumps between synchronization states along the run $N_{J}$. To do this last calculation, I consider the system to be weakly synchronized when one of the $D_{\alpha}$ is less $3 \sigma$, while all the others are much greater than that quantity. A jump happens when the particular value of $\alpha$ for which $D_{\alpha}$ is small, the others being large, changes. This choice is justified by what follows. Moreover, I will note that, in this subsection, the histograms $\Delta N_{\alpha}(D)$ are presented under the form for the functions $f_{\alpha}(D)$ (a)

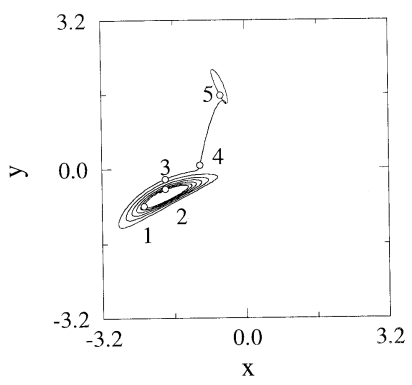

(c)

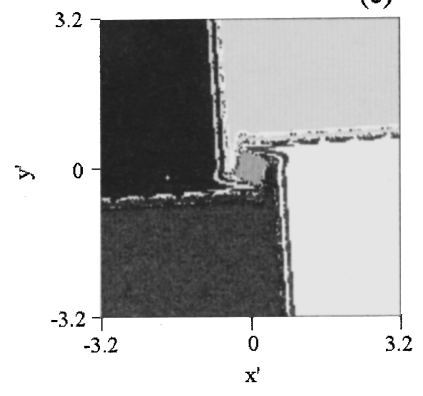

(b)

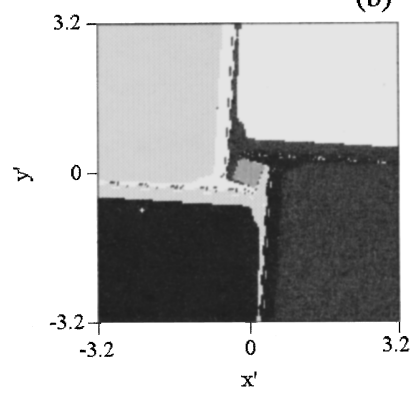

(d)

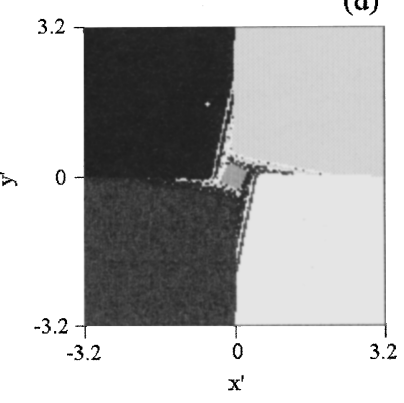

FIG. 12. (a) Drive attractor trajectory, for A3, with indication of the points taken as initial conditions for the calculation of the basins of attraction depicted in this figure and in Figs. 13 and 14. Plots of the basins of attraction of the response to the different behaviors allowed to it when $z$ driving is applied: (b) point 1 , (c) point 3, and (d) point 5 . 
(a)

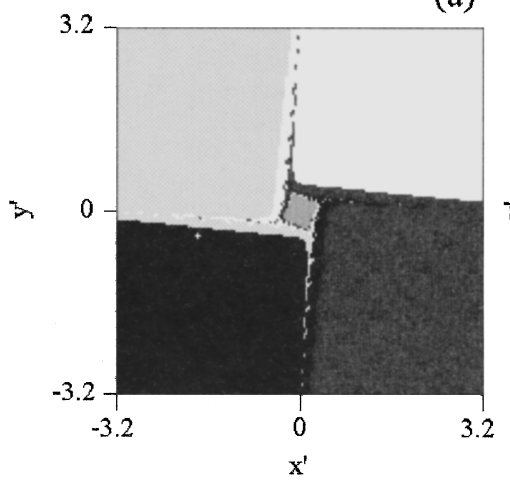

(b)

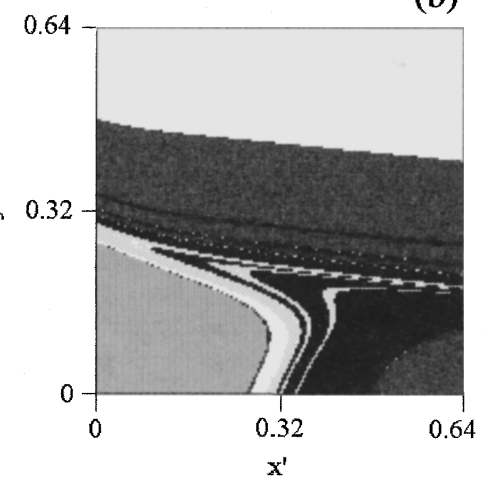

(c)

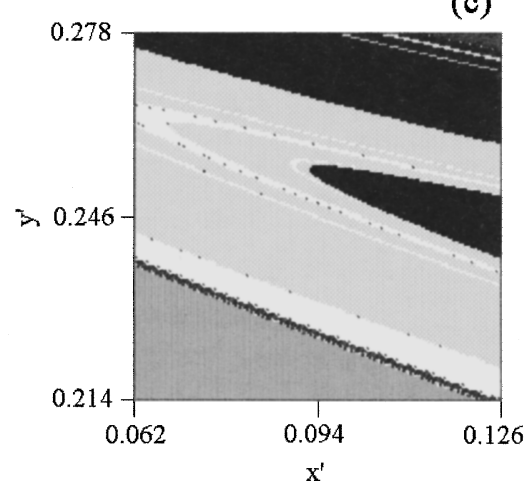

FIG. 13. Plots of successive amplifications of the basin of attraction of the response to the different behaviors allowed for A3. The initial condition for the drive point is given by point 2 in Fig. 11(a). The respective centers of the figures and amplification factors are: (a) $(0,0)$ and $\times 1$, (b) $(0.32,0.32)$ and $\times 10$, and $(\mathrm{c})(0.094,0.246)$ and $\times 100$.

$=\Delta N_{\alpha}(D) / N_{\text {Obs }}$, being $N_{\text {Obs }}$ the number of observations of $D$ in the above time window, for a given $\Delta D$. In each case the value of $\Delta D$ has been chosen according to the range of definition of the function $f_{\alpha}(D)$, to ensure that their shapes are well resolved. The results obtained are independent of the particular choice of $\alpha$ for the basin of attraction of the initial condition.

For A1 I have integrated, together with the above Eqs. (1) for the drive, the following equations for the response:

$$
\begin{gathered}
\dot{x}^{\prime}=-\alpha x^{\prime}+\beta(z+\eta) \sin \left(y^{\prime}\right), \\
\dot{y}^{\prime}=-y^{\prime}+[(z+\eta)-\gamma] x^{\prime},
\end{gathered}
$$

with $\eta$ the above random Gaussian variable. The shape of the histograms $f_{\alpha}(D)$ for $D_{0}(t)$ and $D_{1}(t)$ exhibits the same behavior, no matter in which basin of attraction the initial condition was set. This is illustrated by means of the results for $f_{0}(D)$ and $f_{1}(D)$, at a particular value of $\sigma$, shown for the small $D$ region in Fig. 16(a) and for the large $D$ region in Fig. 16(b). Both distribution functions show the same appearance and are practically put on top of each other despite the statistics implying only a finite number of observations. This is an indication of repeated changes between synchronization states. That is, what we see in these figures, for each $f_{\alpha}(D)$, is the combination of the distribution functions for two different synchronization states: the case when the re- sponse is in the $D_{\alpha} \rightarrow 0$ state [Fig. 16(a)] with the case when the response is in the $D_{\alpha^{\prime} \neq \alpha} \rightarrow 0$ state [Fig. 16(b)]. In the small $D_{\alpha}$ region these functions present a well defined maximum close to zero, which signals a state of weak synchronization, in which the distance between both systems fluctuates around the distance corresponding to this maximum. The large $D_{\alpha}$ regions correspond to the time when the system is in the other synchronization state so that the distance fluctuates around values that are of the same order of magnitude as the attractor size. It is important to notice that the distribution functions for these two states have an overlapping region, as seen in Fig. 16(b), in accordance with the idea that the system can easily jump between synchronization states. However, one has a well defined maximum for the times when generalized synchronization occurs, so that it is possible to compute the dependence of the value of the position of the maxima $\bar{D}$, with $\sigma$. The results, that appear in Fig. 16(c), can be accurately fitted by a potential law of the type $\bar{D}_{\alpha}(\sigma)=A \sigma^{b}$, being $A=0.419 \pm 0.015, b=0.999 \pm 0.005$, and the correlation coefficient 0.99994 . The dependence of the number of jumps between synchronization states $N_{J}$ with the amplitude of the noise $\sigma$ is displayed in Fig. 16(d). The function $N_{J}(\sigma)$ can be approximated by a law of the type $N_{J}(\sigma)=A \sigma^{b}$ with $A=1.10 \times 10^{6} \pm 1.7 \times 10^{5}$ and $b=1.039$ \pm 0.021 , being the correlation coefficient 0.99983 . As the interval of the variation of the $z$ signal is 6.37 wide, we see that above a noise as small as $1.6 \times 10^{-6}$ of the amplitude of (a)

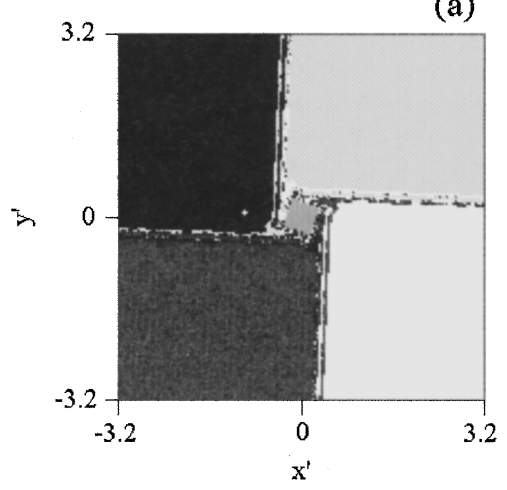

(b)

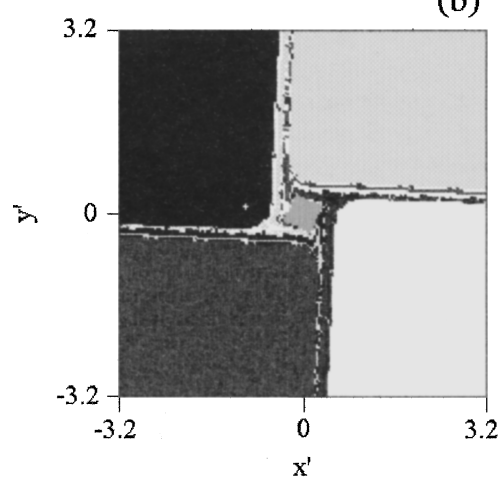

(c)

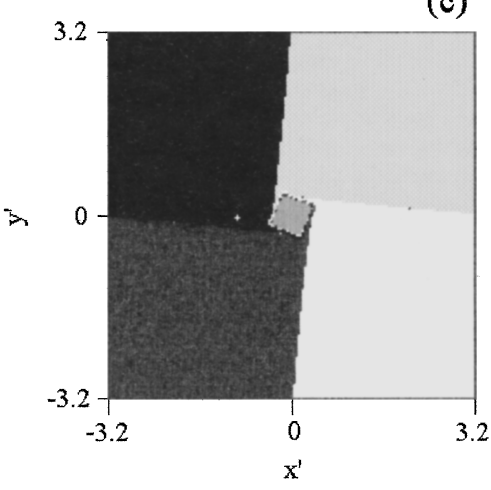

FIG. 14. Plots of the basin of attraction corresponding to the attractor A3 for almost the same initial condition of the drive [point 4 of Fig. 12(a)], and different values of the parameter $\sigma:$ (a) 16.0, (b) 14.0, and (c) 12.0. 
(a)

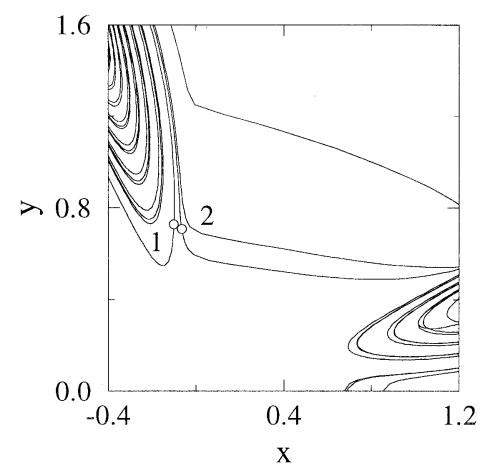

(b)

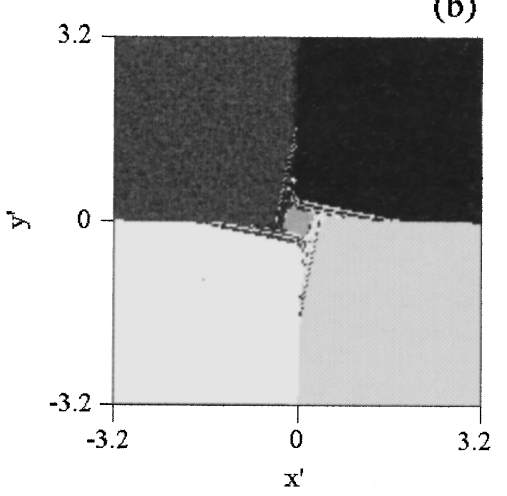

(c)

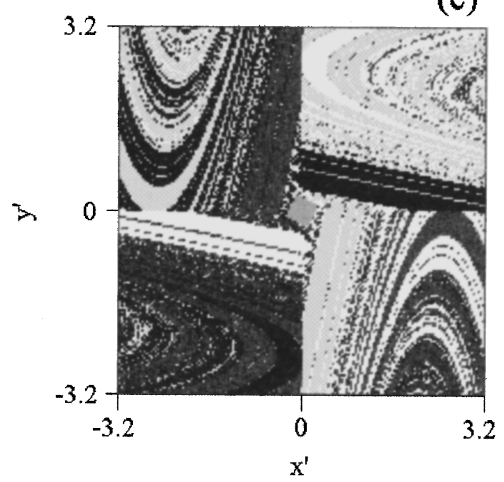

FIG. 15. (a) Drive attractor trajectory with indication of the points taken as initial conditions for the calculation of the basins of attraction depicted in this figure; and, plots of the basins of attraction of the response to the different behaviors allowed to it when $z$ driving is applied: (b) point $1,(\mathrm{c})$ point 2 .

this signal we still see jumps between synchronization states (at this level of noise only nine jumps were observed in the time window studied). Anyway, the extrapolation of $\bar{D}_{\alpha}(\sigma)$ and $N_{J}(\sigma)$ to $\sigma=0$ indicates that, when no noise is present, the system stays in only one of the synchronization states available. The effects of adding noise is to weaken the synchronization and to introduce a rate of jumps between synchronization states. These effects become more and more intense as the level of noise is increased until synchronization is completely lost.

For A2 I have integrated, together with the above Eqs. (2) for the drive, the following equations for the response:

$$
\begin{gathered}
\dot{x}^{\prime}=y^{\prime}+A \sin \left(\Omega y^{\prime}\right), \\
\dot{y}^{\prime}=-y^{\prime}-[(z+\eta)-R] x^{\prime},
\end{gathered}
$$

with $\eta$ the above random Gaussian variable. One must note that these equations also describe the behavior of the system under a fluctuating $R$ parameter for the response, which in a physical situation would stand for a response system in contact with a fluctuating environment. In the following discussion I will assume the noisy drive signal picture, however the translation to the fluctuating $R$ case is straightforward. Figures $17(\mathrm{a})$ and $17(\mathrm{~b})$ show, respectively, the shape of the distribution functions $f_{1}(D)$ and $f_{0}(D)$ for three different values of $\sigma$. The initial condition for this particular picture was in the $D_{1} \rightarrow 0$ basin of attraction. Those plots indicate that both distances fluctuate inside intervals that are well separated; i.e., negligible overlap between $f_{\alpha}(D)$ functions at different synchronization states. For $f_{1}(D)$, this interval is narrow and close to zero, while for $f_{0}(D)$ it is wide and far from zero. Moreover, the values for $f_{1}(D)$ can be scaled in a single curve when the distance is divided by the strength of the noise. For values of $\sigma$ below 0.8 , the average value of the distance for the weakly synchronized state $\left\langle D_{1}\right\rangle$ is small and well defined. I have verified that for a size of the sample
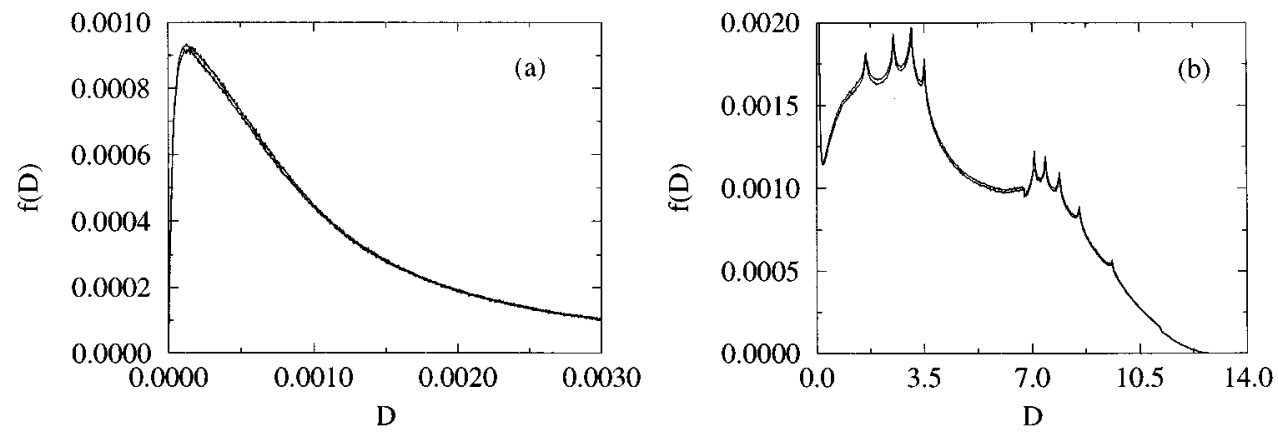

FIG. 16. Shape of the histograms $f_{\alpha}(D)$ for the distances $D_{0}(t)$ and $D_{1}(t)$ for attractor A1 under a level of noise of $\sigma=0.0003$ in (a) the small distances region, and (b) the large distances region. To have the function shapes well defined, different intervals $\Delta D$ have been used for the calculations in (a) and (b). (c) Dependence of the positions of the maxima of the distributions with the strength of the noise for the weakly synchronized state. (d) Dependence of the number of synchronization state changes with the strength of the noise. In (c) and (d), the squares are for numerical results and the lines for their least squares fits. 

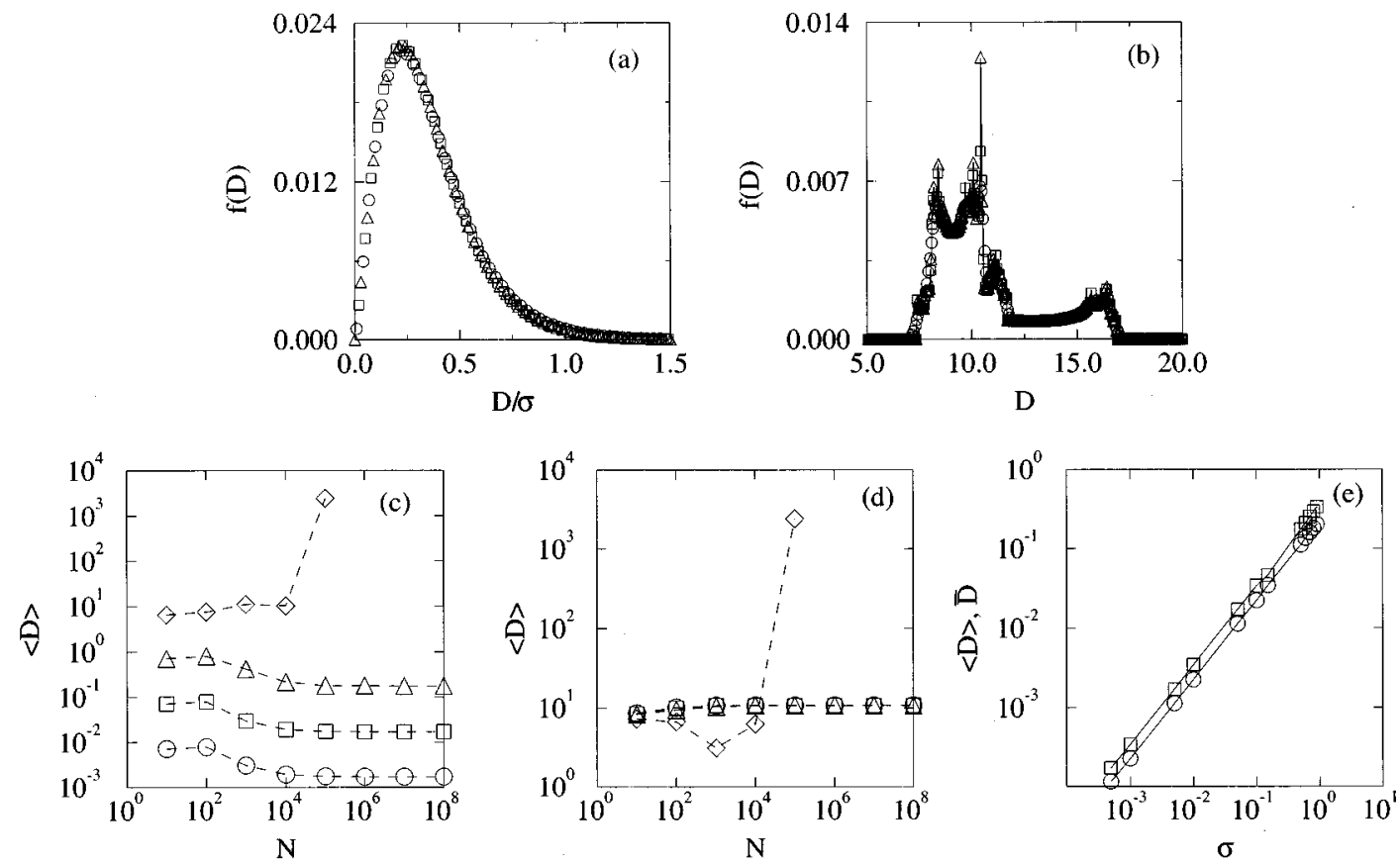

FIG. 17. Shape of the histograms $f_{\alpha}(D)$ for attractor A2 under a level of noise of $\sigma=0.005$ (circles), $\sigma=0.05$ (squares), and $\sigma=0.5$ (triangles): (a) $f_{1}(D)$ and (b) $f_{0}(D)$ for initial conditions in the $D_{1} \rightarrow 0$ basin of attraction. To have the function shapes well defined, different intervals $\Delta D$ have been used for the calculations in (a) and (b). Proof of the stability of the average value of $D$ for: (c) $D_{1}$ and (d) $D_{0}$. Circles, squares, and triangles have the same meaning as in (a) and (b), diamonds are the results for $\sigma=5.0$. (e) Dependence of the positions of the maxima of the distributions, $\bar{D}$, (squares), and the average distance for values of a weakly synchronized state, $\langle D\rangle,($ circles), with the strength of the noise $\sigma$. The lines are least squares fits.

large enough, its numerical value does not change when this size is increased [see Fig. 17(c)]. In the same way the average value of the distance $D_{0},\left\langle D_{0}\right\rangle$, is well defined too, but of the same size as the attractor [Fig. 17(d)]. When the initial condition is chosen in the $D_{0} \rightarrow 0$ basin of attraction these comments would be the same but swapping indexes 0 and 1 . I have found that for $\sigma \leqslant 0.8$, in the time window studied there are no jumps between the different synchronized and nonsynchronized states available. Moreover, the relation between $\langle D\rangle$ and $\sigma$, for the weakly synchronization state, can be accurately fitted by a law of the type $\langle D\rangle=A \sigma^{b}$, with $A=0.357 \pm 0.008, b=1.007 \pm 0.002$, and the correlation coefficient 0.99998 [see Fig. 17(e)]. A similar result is obtained when $\bar{D}$ is studied; in this case, it is $A=0.226 \pm 0.001$, and $b=0.999 \pm 0.001$ and the correlation coefficient is 0.99986 . The concordance is clear between the most probable and average distances. The interval of variation of the $z$ signal is 6.57 wide; then, below a noise as large as 12 percent of the amplitude of the signal, we can have the response in one of the generalized synchronization states, evolving at an average generalized distance of the drive of the same order of magnitude than the noise applied, at least in the time window studied here. For values of $\sigma$ above 0.8 the response jumps between the several basins of attraction available. Then, sooner or later, it falls into the basin of divergent initial conditions, and the value of $\left\langle D_{\alpha}\right\rangle$ becomes a quantity that increases when the interval where the time average is performed is increased [see Fig. 17(c) and Fig. 17(d)].

For A3 I have integrated, together with the above Eqs. (3) for the drive, the following equations for the response:

$$
\begin{aligned}
\dot{x}^{\prime}= & \epsilon\left(x^{\prime}, y^{\prime}\right)\left[-\alpha x^{\prime}+\beta(z+\eta) \sin \left(y^{\prime}\right)\right] \\
& +\delta\left(x^{\prime}, y^{\prime}\right)\left\{-x^{\prime}+[\gamma-(z+\eta)] y^{\prime}\right\}, \\
\dot{y}^{\prime}= & \epsilon\left(x^{\prime}, y^{\prime}\right)\left\{-y^{\prime}+[(z+\eta)-\gamma] x^{\prime}\right\} \\
& +\delta\left(x^{\prime}, y^{\prime}\right)\left[-\alpha y^{\prime}-\beta(z+\eta) \sin \left(x^{\prime}\right)\right],
\end{aligned}
$$

with $\eta$ the above random Gaussian variable. The effect of noise is a generalization of the results described for A1 to a $C_{4}$ symmetry case. This is illustrated in Figs. 18(a) and 18(b) where the $f_{\alpha}(D)$ functions for the four generalized distances implied are practically indistinguishable from each other, despite the limited statistics used. As in the two previous cases, weak synchronization is obtained with distributions functions peaked at a value close to zero for the synchronized state and broad distributions for the nonsynchronized. Although, these distributions show overlaps with increasing noise, one still has a well defined maxima at $\bar{D}$ that can be studied as a function of the noise strength [Fig. 18(c)]. Moreover, as in A1, jumps between synchronization states are present and its number increases with the noise strength [Fig. 18(d)]. The laws describing these events are potential laws. For the position of the maxima $\left[\bar{D}_{\alpha}(\sigma)=A \sigma^{b}\right]$ one has $A=0.223 \pm 0.005$ and $b=0.999 \pm 0.002$ with correlation coefficient 0.999 97. And for the number of jumps $\left[N_{J}(\sigma)=A \sigma^{b}\right], \quad A=3.93 \times 10^{4} \pm 2.6 \times 10^{3}$ and $b=0.454$ \pm 0.005 with a correlation coefficient of 0.99923 . Being the range of variation of the $z$ signal 3.23 wide, we obtain that above a noise as small as $3.1 \times 10^{-9}$ of the amplitude of the 

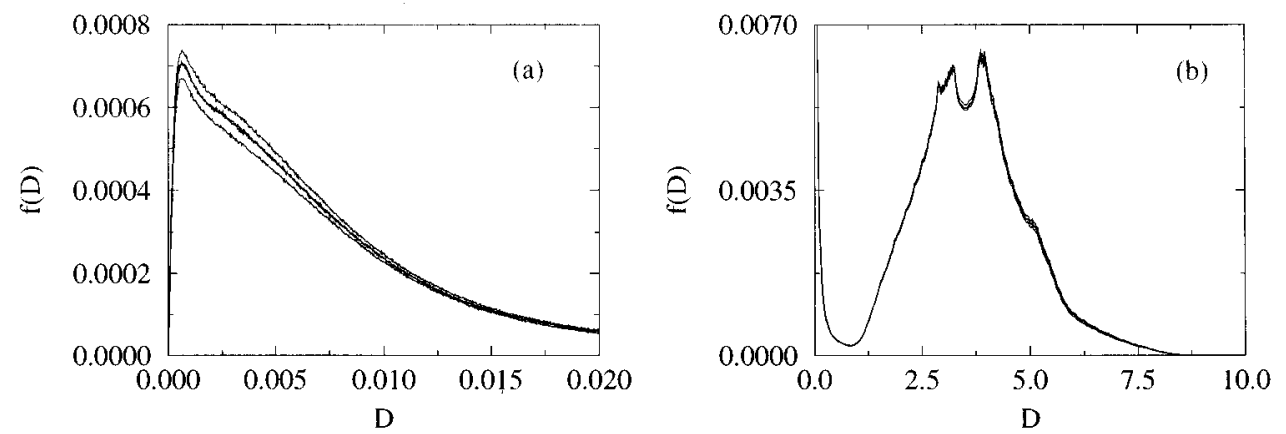

FIG. 18. Shape of the histograms $f_{\alpha}(D)$ for the distances $D_{0}(t), D_{1}(t), D_{2}(t)$, and $D_{3}(t)$ for attractor A3 under a level of noise of $\sigma=0.003$ in (a) the small distances region and (b) the large distances region. To have the function shapes well defined, different intervals $\Delta D$ have been used for the calculations in (a) and
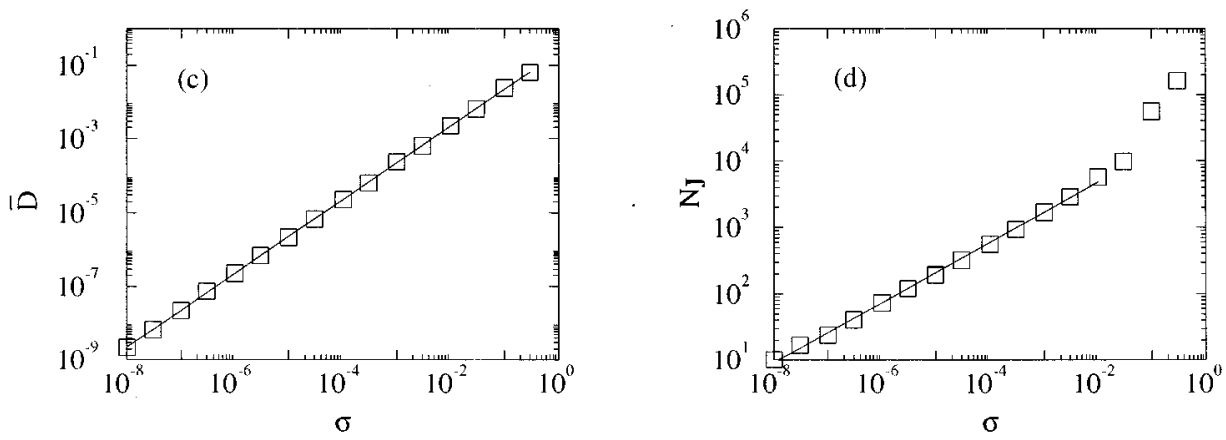
(b). (c) Dependence of the position of the maxima of the distributions with the amplitude of the noise for the weakly synchronized state. (d) Dependence of the number of synchronization state changes with the strength of the noise. In (c) and (d), the squares are for numerical results and the lines for their least squares fits.

signal we still observed jumps between synchronization states in the time window considered (only ten jumps at this level of noise).

In general, the two effects of noise expected in Sec. II are observed in the computer experiments: weak synchronization and changes between synchronization states. These effects become more important with the strength of the noise. The mathematical laws followed are potential, so that in the free noise cases there is full synchronization with no jumps between synchronization states. A consequence of this is that one can have one of the $D_{\alpha}(t) \approx 0$ and no jumps between states in a time window as large as desired when the noise level is low enough. Moreover, the results for A2 illustrate that, when the topology of the system is adverse for the jumps between synchronization states, except for extremely large fluctuation that are very improbable, one can expect only a weakening of the synchronization with no jumps between synchronization states, even for quite large perturbations. Anyway these results sustain the idea that the generalized synchronization states introduced here are not artifacts of the computer, but robust situations that may be observable in an experiment.

\section{SUMMARY AND CONCLUSIONS}

In this article, I have shown how the symmetries in the equations of a nonlinear dynamical system led to an extension of the idea of synchronization of two chaotic systems, introduced by Pecora and Carroll, to an interesting situation in which the system being controlled may synchronize in more than just one way with the drive system. This represents an enrichment of the possibilities of synchronization because, for a given system, there emerges a variety of states of the response, physically different among them, but in synchrony with the drive. Then, the space of initial conditions is divided in basins of attraction to the different synchroniza- tion states available — and, eventually, to nonsynchronization ones. Moreover, two different responses to external perturbations are possible: weakening of the synchronization and jumping between synchronization states.

The phenomenon has been illustrated by means of a computer simulation study of three mathematical models of chaotic systems, two of them bearing inversion symmetry and one belonging to a large symmetry group $\left(C_{4}\right)$. Moreover, in some parts of the computational part of the paper, different sets of parameters have been considered. From this computer simulation results it follows that:

(1) When we deal with symmetric systems, it is possible to have synchronization states of types different that the usual. These can be described by means of convenient generalized distances, that decay to these states in an oscillatory exponential fashion.

(2) The shapes of the basins of attraction to the different synchronization states available are specific to each particular system. Even different systems having the same type of symmetry will have patterns that differ qualitatively. However there are some regularities: (i) the basins display symmetries reminiscent to those of the dynamical system to which they belong, and (ii) they change smoothly under the change of the parameters of the system. Moreover, it has been found that the boundary basins may be fractal in many cases.

(3) External noise has two effects: it weakens the synchronization and gives rise to jumps between synchronization states. The dependence with the strength of the noise for both effects can be described by means of potential laws. The weakening of the synchronization presents a behavior common to all systems characterized by a well defined characteristic distance. The jumping behavior is strongly dependent on the topology of the system. In particular, for attractors with toroidal topology, even under relatively large perturbations, one can expect to observe only a weakening of 
the synchronization with no jumps between synchronization states.

Inside the frame of the general properties just mentioned, each particular nonlinear system will have its own characteristic properties, i.e., generalized synchronization states available, shapes of the basins of attraction, and response to external perturbations. This should not be a surprise because an important characteristic of nonlinear systems is the richness of behaviors available. In fact, the work presented here does not exhaust the possibilities of study of synchronization of symmetric chaotic systems, it only draws attention to their interesting possibilities. Anyone willing to work on the synchronization of a particular symmetric chaotic system should perform a particular study of that system.

Possible extensions of the present work may include the study of the synchronization of symmetric maps, instead of flows. Prospective candidates for such a study will be the maps with the symmetry of an $m$ gon in the plane introduced by Chossat and Gollubitsky in their work on symmetryincreasing bifurcations of chaotic maps [23]. Other possible candidates for such studies will be models that are more that pure mathematical entities and represent real physical systems. An example of such models may be the network of oscillators with the symmetries of an equilateral triangle used by Ashwin to model coupled oscillating neurons [24].

The ideas presented here have been tested only in numerical experiments with mathematical models. As it has been found that the synchronization must be robust against weak external noise and small changes in the parameters of the system, there is founded hope that it will be observable in experiments with real systems. This observation will be the most important step forward in the issue of multiple synchronization states and symmetry. It seems to this author that electronic circuits will be the easiest way to realize such experimental approach. This is because the usual Pecora and Carroll synchronization has been observed in electronic circuits $[4,5]$, and these are versatile systems able to be tailored for specific purposes. In particular, the use of nonlinear elements with piece-wise constitutive relations [25] will be especially useful to design systems like the one defined by Eqs. (3).

At a more speculative level, one might think the bistability (or multistability) of the kind of systems presented here to be of interest in relation to problems in communication and control theory, or in neural systems science. For example, patterns resulting from a set of response systems under the same drive, as those shown in Sec. III, are objects containing information. In the frame of the emerging view of neurons and neural nets as dynamical chaotic systems [26,27] this might be of interest to study processes of storage and retrieval of information.

Finally, I will note that the results in the present paper provide additional illustration of two important results reported by other authors. These are: (i) the observation of systems with negative conditional Lyapunov exponents, in which not all the initial conditions of drive and response give rise to synchronization, as suggested by Badola, Tambe, and Kulkarni [3], and (ii) the report of particular examples of generalized synchronization behaviors introduced in more general terms by Amritkar and Gupte [13] and by Rulkov et al. [14].

\section{ACKNOWLEDGMENTS}

I gratefully acknowledge financial support from DGICIT (project PB93-0780) and partial computational support from CESCA.
[1] L. M. Pecora and T. L. Carroll, Phys. Rev. Lett. 64, 821 (1990).

[2] L. M. Pecora and T. L. Carroll, Phys. Rev. A 44, 2374 (1991).

[3] P. Badola, S. S. Tambe, and B. D. Kulkarni, Phys. Rev. A 46, 6735 (1992).

[4] T. L. Carroll and L. M. Pecora, IEEE Trans. Circuits and Systems 38, 453 (1991)

[5] L. O. Chua, L. Kocarev, K. Eckert, and M. Itoh, Int. J. Bif. Chaos 2, 705 (1992).

[6] L. Kocarev, K. S. Halle, K. Eckert, L. O. Chua, and U. Parlitz, Int. J. Bif. Chaos 2, 709 (1992).

[7] K. Murali and M. Lakshmanan, Phys. Rev. E 48, R1624 (1993).

[8] T. L. Carroll and L. M. Pecora, Physica D 67, 126 (1993).

[9] T. L. Carroll, IEEE Trans. Circuits and Systems 42, 105 (1995).

[10] K. Pyragas, Phys. Lett. A 170, 421 (1992).

[11] N. F. Rulkov, L. S. Tsimring, and H. D. I. Abarbanel, Phys. Rev. E 50, 314 (1994).

[12] J. M. Kowalski, G. L. Albert, and G. W. Gross, Phys. Rev. A 42, 6260 (1990).

[13] R. E. Amritkar and N. Gupte, Phys. Rev. A 44, R3403 (1991).
[14] N. F. Rulkov, M. M. Sushchik, L. S. Tsimring, and H. D. I. Abarbanel, Phys. Rev. E 51, 980 (1995).

[15] O. E. Rössler, Phys. Lett. A 57, 397 (1976).

[16] E. N. Lorenz, J. Atmos. Sci. 20, 130 (1963).

[17] J. A. Jacobs, Phys. Rep. 26, 183 (1976).

[18] F. C. Moon, Chaotic Vibrations (Wiley, New York, 1987).

[19] H. G. Schuster, Deterministic Chaos (VCH Verlagsgessellschaft mbH, Weinheim, 1988).

[20] G. Benettin, L. Galgani, A. Giorgilli, and J.-M. Strelcyn, Meccanica 15, 21 (1980).

[21] I. Shimada and T. Nagashima, Prog. Theor. Phys. 61, 1605 (1979).

[22] H. Haken, Phys. Lett. A 94, 71 (1983).

[23] P. Chossat and M. Gollubitsky, Physica D 32, 423 (1988).

[24] P. Ashwin, Nonlinearity 3, 604 (1990).

[25] L. O. Chua, C. W. Wu, A. Huang, and G.-Q. Zhong, IEEE Trans. Circuits and Systems 40, 745 (1993).

[26] Coupled Oscillating Neurons, edited by J. G. Taylor and C. L. T. Mannion (Springer-Verlag, London, 1992), pp. 21-40.

[27] P. K. Das II, W. C. Schieve, and Z. J. Zeng, Phys. Lett. A 161, 60 (1991). 